\title{
Probe and Emission Spectrometry Diagnostics in Hollow Cathode Magnetron
}

\author{
N. P. Poluektov, Yu. P. Tsar'gorodsev, I. I. Usatov, \\ A. G. Evstigneev, I. A. Kamyschov \\ Department of Physics, Faculty of Electronics, Moscow State Forest University, Mytischi, Russia \\ Email: poluekt@mgul.ac.ru
}

Received August 10, 2012; revised September 12, 2012; accepted September 19, 2012

\begin{abstract}
This paper deals with the characterization of an ionized physical vapor deposition (IPVD) by means of hollow cathode magnetron. Langmuir probe, optical emission spectroscopy measurements were used to study a mechanism for the production of excited argon and copper atoms and ions. The kinetic processes of excitation were considered and the main processes were determined using results of measurements. The pressure range is $0.5-10$ mTorr with $1-5 \mathrm{~kW}$ discharge power. Plasma parameters such as electron densities and temperatures, electron energy distribution function, plasma space and floating potentials as a function of the position, pressure and power in the growth chamber were measured. The plasma density is up to $10^{12} \mathrm{~cm}^{-3}$ at $20 \mathrm{~cm}$ from the magnetron for $10 \mathrm{mTorr}$.
\end{abstract}

Keywords: Hollow Cathode Magnetron; Ionized Physical Vapor Deposition

\section{Introduction}

The hollow cathode magnetron (HCM) is new type of a source of plasma for films deposition using of atoms and ions of metal. Feature of this discharge is high density plasma (more than $10^{12} \mathrm{~cm}^{-3}$ at pressure a few millitorrs) created in big $\left(10^{3} \mathrm{~cm}^{3}\right)$ volume, low $(10-50 \mathrm{eV})$ and easily changeable energy of the ions arriving at the substrate. The characteristic difference between this technique and conventional approaches is that high fraction of the sputtered material is ionized, while in traditional magnetron sputtering, the sputtered species are almost exclusively neutral. The ionized physical vapor deposition (IPVD) method is increasingly used to deposit diffusion barriers and copper seed layers materials into highaspect ratio vias and trenches for microelectronics fabrication [1-5]. Ionized metal plasmas have been also used to produce nanosize interlayers and graded structures by intermixing of condensing ions and substrate. Metal plasmas are often used in deposition of nanosized compound multilayers that can undergo phase changes at elevated temperatures [6,7]. Film deposition in this discharge is accompanied by the streams of low-energy ions that allow to receive a film with unique properties.

Several techniques have been developed for obtaining an ionized growth flux; the plasma may be generated, for example, by electron cyclotron resonance [8] or by inductively coupled radio-frequency (rf) power [3-7].
HCM uses a single de power supply to both sputter and ionize the target material unlike other IPVD tools which use secondary inductively coupled or ECR plasma sources for ionization of sputtered atoms. The quality of the deposited films depends on the quantity and energy of the particle flux and substrate temperature. In the HCM these values differ greatly from those in the convectional magnetron. In a conventional magnetron main contribution to the heat flux on the substrate is made by atoms of the target and buffer gas. In HCM main contribution is produced by ions of the buffer gas and metal. Such ion assistance allows the deposition of high quality films on complex shaped substrates. Only a few papers have been published which deal with investigation HCM $[1,2,9,10]$. The purpose of this paper is to study a mechanism for the production of excited argon and copper atoms and ions. For this the spatial distribution of plasma parameters in hollow cathode magnetron was studied using probe and optical emission spectroscopy techniques.

\section{Experimental Apparatus}

Figure 1 presents our experimental set-up. The cathode consists of a cup-shaped $\mathrm{Cu}$ target $(8 \mathrm{~cm}$ i.d. and $7 \mathrm{~cm}$ long) from which plasma diffuses into a reactor $(35 \mathrm{~cm}$ diameter, $55 \mathrm{~cm}$ length). The chamber was pumped to base pressure $5 \times 10^{-6}$ Torr using a turbomolecular pump. 


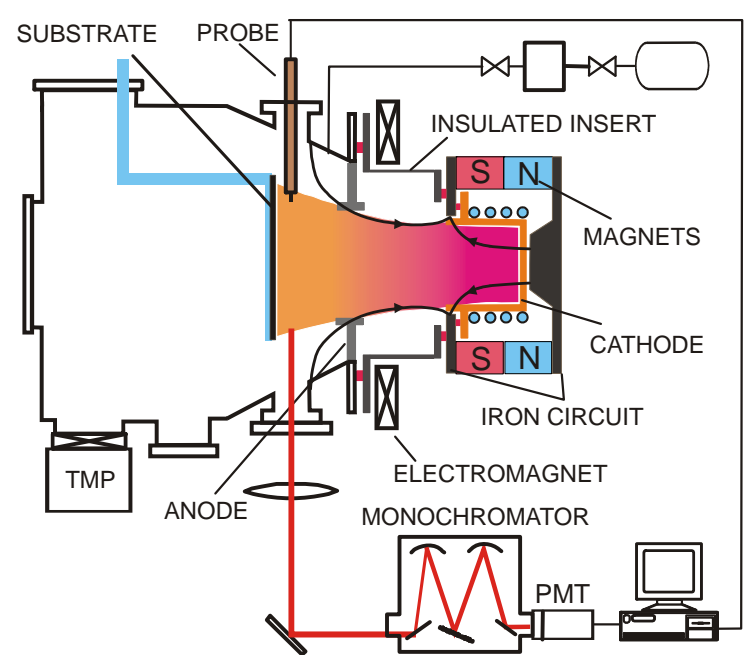

Figure 1. Schema of the experimental set-up.

Argon is used as the buffer gas.

Pressure was within range of 0.5 - 10 mTorr. Gas flow is provided by a gas flow controller. The HCM is powered with inverter source up to $12 \mathrm{~kW}(20 \mathrm{~A}, 600 \mathrm{~V})$. The magnetic field with maximum of 800 Gs is produced by twelve columns of Nd-Fe-B magnets $18 \times 20 \times 120$ $\mathrm{mm}^{3}$ in size surrounded the target with ring iron flanges on the end. The downstream of the HCM is located the electromagnet that creates a magnetic field of opposite direction to the field of the permanent magnets. As a result magnetic field is directed along a sidewall surface of the magnetron and has a cusp at the mouth of the cathode. Magnetic field captures secondary electrons emitted from the cathode, which produce an ionization of the buffer gas and sputtering atoms of the target. Crossed ExB fields cause electron drift in an azimuthal direction, in result inside the hollow cathode plasma of high density $\left(>10^{13} \mathrm{~cm}^{-3}\right)$ is created. The target utilization in such cathode is higher than in the magnetron with flat cathode. Figure 2 shows the change in the thickness of the cathode measured along its length. The zone of erosion occupies almost all cylindrical part.

Outside of the hollow cathode there is the region where the magnetic field strength is equal to zero. This area separates the plasma that exists in the hollow cathode from plasma which flows toward the substrate. Those electrons and ions which have initial axial velocities are capable to leave the hollow cathode and to be distributed to a substrate. The plasma stream has a core with diameter of about $4 \mathrm{~cm}$ at a distance of $20 \mathrm{~cm}$ from a magnetron. For expansion of plasma stream and creation of more homogeneous radial distribution is used the electromagnet that creates a diverging magnetic field outside the magnetic null region. The electrically insulated insert is located between the cathode and plasma chamber to create a potential difference with respect to

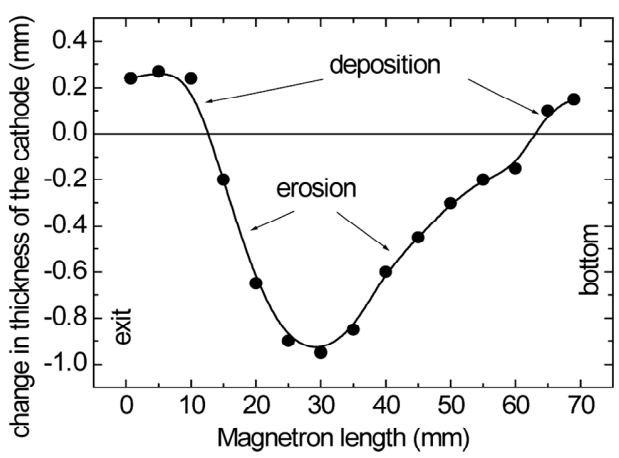

Figure 2. Structures of the target erosion.

HCM. The electron temperature, electron energy distribution function, ion density, floating and plasma potentials were determined from probe measurements. The probe tip was made of a tungsten wire $0.35 \mathrm{~mm}$ in diameter and $5 \mathrm{~mm}$ long. The probe was located at a distance of $20 \mathrm{~cm}$ from exit of a magnetron and $3 \mathrm{~cm}$ before a substrate. The substrate was isolated from chamber wall. It should be noted that probe measurements in this plasma are not a simple task due to metal deposition on the probe. Discharge power is kWatts and metal flux is large. In the article [9] his issue is considered in details.

Therefore we created a system for rapid record of the probe characteristics. The I-V characteristics were recorded with the help PCI card National Instruments NI6221 with a 16 bit ADC, a 16 bit DAC and multiplexer. The ADC and DAC were connected to a probe via isolated modules. The DAC voltage was increased by self-made powerful voltage amplifier (with an output voltage range from -80 to $+80 \mathrm{~V}$ at an output current of up to $800 \mathrm{~mA}$ and a voltage rise time of up to $10 \mathrm{~V} / \mathrm{s}$ ). The I-V characteristic includes up to 640 pairs. In dense plasma the number of points is less $(420-450)$ as the voltage range is limited to $15-20 \mathrm{~V}$ due to large electron saturation current. To improve the accuracy of measurements, each pair of current-voltage points is obtained by averaging of the set of 10 data points. The time required to obtain one $\mathrm{I}-\mathrm{V}$ curve is about $2 \mathrm{~s}$. The program of data processing is written in MatLAB language. At first the data smoothing by B-splines is made and then the plasma potential and electron energy distribution function (EEDF) are calculated from the second derivative. The need for this procedure is due to the fact that experimental data have large noises caused by fluctuations of plasma. In the plasma of our discharge the ratio of probe radius to Debye radius is about of 10 , therefore analytical Langmuir theory is not applied for the probe analysis. For calculation of electron density is used the parametrization of the Laframboise theory [11]. The algorithm used in the program is the development of the method described in [12]. The electron temperature is defined as average temperature: 


$$
T_{e}=\frac{2}{3 k} \frac{\int_{0}^{\infty} E f(E) \mathrm{d} E}{\int_{0}^{\infty} f(E) \mathrm{d} E}
$$

where $E, k, f(E)$ are the energy of the electrons, the Boltzmann constant and the energy distribution function of electrons respectively.

Plasma emission was monitored through windows located at $20 \mathrm{~cm}$ downstream the magnetron in the conical part of the chamber. To prevent quartz windows from metal deposition two $5 \mathrm{~cm}$ tubes with $1.5 \mathrm{~cm}$ diaphragm are located inside the chamber, providing between them $15 \mathrm{~cm}$ length trough plasma.

Spectra of plasma emission were measured by a grating monochromator (1200 lines $\mathrm{mm}^{-1}$, inverse dispersion $2.4 \mathrm{~nm} / \mathrm{mm}$ ) equipped with a photomultiplier tube (PMT).

\section{Results and Discussion}

Figure 3 shows current-voltage (I-V) characteristics of the magnetron discharge for various pressures. The (I-V) characteristics are well approximated by the relationship $I=k V^{n}$ with $\mathrm{n}=7-10$. With increasing pressure the discharge voltage decreases for the same currents, it is connected to growth of plasma density. The probability of ionization is proportional to neutral particles density and this effect is greater than the reduction of electron temperature, which results in opposite effect.

Figure 4 shows an effect of a magnetic field of an electromagnet on the radial plasma characteristics. The increase of a magnetic field of the electromagnet results in growth of uniformity of a stream and to a decrease of plasma density. At discharge power of $3 \mathrm{~kW}$ and pressure of 10 mTorr plasma density on an axis decreases from $7.2 \times 10^{11} \mathrm{~cm}^{-3}$, when electromagnet is turned off, to $3 \times 10^{11} \mathrm{~cm}^{-3}$ when electromagnet current is equal to 1.2 A. Let's note also, that energy of ions on the isolated substrate, equal $e\left(V_{s}-V_{f}\right)$ does not exceed $20 \mathrm{eV}$.

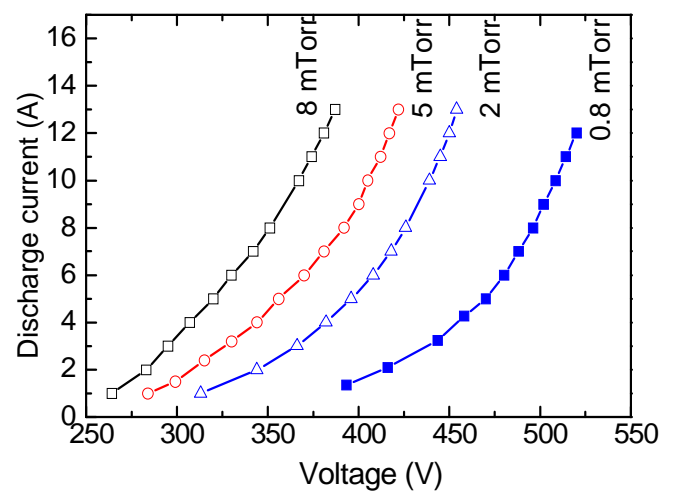

Figure 3. Current-voltage characteristics of the HCM for various pressures.

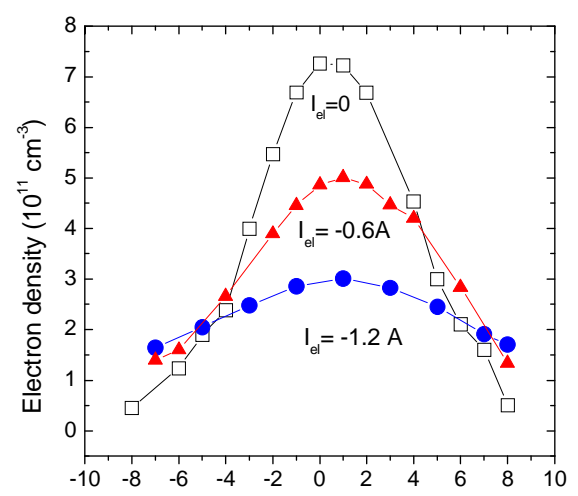

(a)

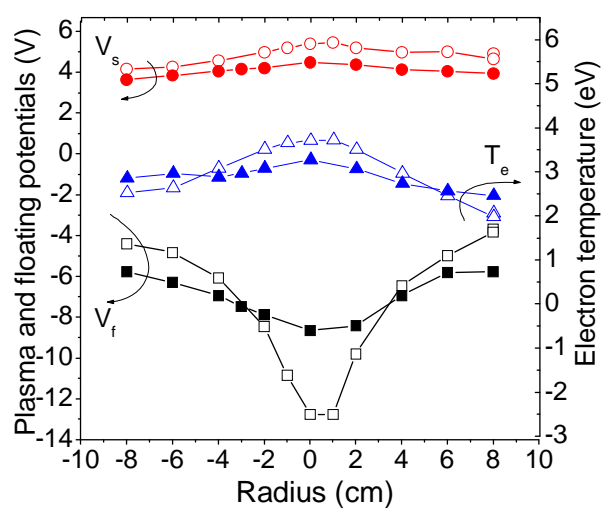

(b)

Figure 4. Radial profiles of: (a) The electron density $N_{e}$; (b) Electron temperature $T_{e}$, floating $V_{f}$ and plasma $V_{s}$ potentials. $20 \mathrm{~cm}$ from the target. $p=10 \mathrm{mTorr}, 50 \mathrm{sccm}, W=3$ kWatt. Open symbols: $I_{\mathrm{el}}=0 \mathrm{~A}$, solid symbols: $I_{\mathrm{el}}=-0.6 \mathrm{~A}$.

Mean electron temperature, floating and plasma potentials decrease with electromagnet current increase. Probably, it occurs owing to growth of the electron losses on excitation and ionization of atoms at increase of cross section of a stream. Nevertheless, the high values of electron density and temperature on this distance allow effectively ionize the sputtered metal atoms for a way from the target to the substrate. It should be noted, that plasma density inside of the cathode changes very little, as I-V characteristics of the discharge depend poorly on a current of an electromagnet. Thus, change of plasma density occurs outside of the cathode where magnetized electrons move along divergent magnetic field lines of the electromagnet on lateral walls of the chamber and by ambipolar diffusion pull behind itself ions.

Effect of the magnetron power on plasma parameters on the discharge axis is shown in Figure 5. The plasma density (Figure 5(a)) grows almost linearly with a power up to a level of $2 \mathrm{~kW}$, then slope decreases. Such behavior can be explained by a decrease of the local argon density due to heating by sputtered copper atoms. Plasma and floating potentials, average electron temperature depend poorly on a power (Figure 5(b)). Note the mean 


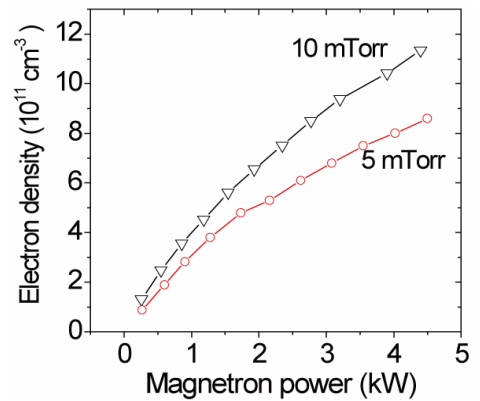

(a)

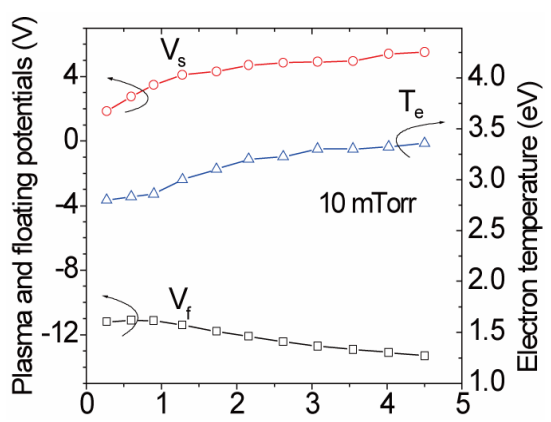

(b)

Figure 5. (a) Electron density; (b) Plasma potential $V_{s}$, floating potential $V_{f}$, and mean electron temperature $T_{e}$; as function of the magnetron power. $I_{\mathrm{el}}=-0.6 \mathrm{~A} .20 \mathrm{~cm}$ from the target.

electron temperature increases with a magnetron power. This fact is not evident for high power IPVD discharges. In $[3-5,9,10]$ the electron temperature decreases for high magnetron current due to very large number of sputtered metal atoms. The energy thresholds for electron impact excitation and ionization of metal atoms $(<8 \mathrm{eV})$ are much lower than those for argon. As a result, metal atoms act as energy absorbers in the discharge, preventing electrons from reaching energies as high as in pure argon discharge. Different results can be explained as follow. In [9] discharge pressure was in the range of $30-50$ mTorr and mean electron temperature has a maximum of $1.5 \mathrm{eV}$. Consequently the number of electrons with energy greater than $8 \mathrm{eV}$ was much less than at pressure 5 10 mTorr with the electron temperature $3-4 \mathrm{eV}$. So the lack of these electrons reduces the mean electron temperature when the number of metal atoms strongly increases.

Figure 6 shows the plasma potential $V_{s}$, floating potential $V_{f}$, and electron temperature as function of a distance from the magnetron at pressures of 2 and 5 mTorr. Near the target plasma density rises with growth of a pressure. Electrons lose its energy in collisions and at a distance of $30 \mathrm{~cm}$ electron density for 5 mTorr becomes less than the one for 2 mTorr. Nevertheless at that distance plasma density exceeds $10^{11} \mathrm{~cm}^{-3}$.

Figure 7 presents on the logarithmic scale the electron

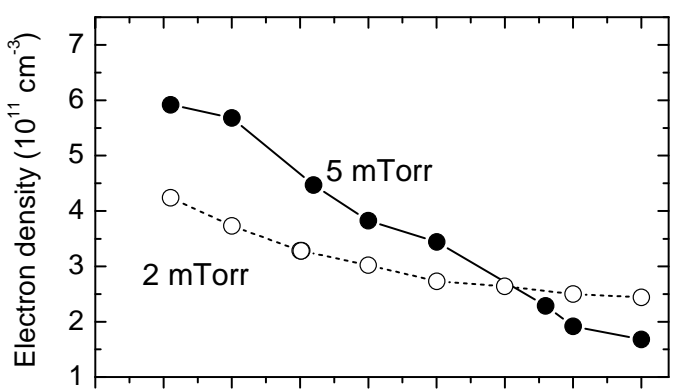

(a)

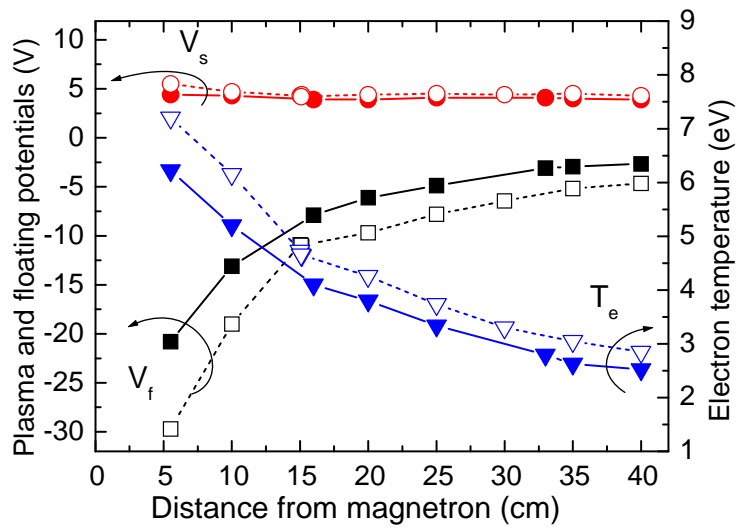

(b)

Figure 6. Axial distribution from the target exit of (a) Electron density $N_{e}$; (b) Electron temperature $T_{e}$, plasma $V_{s}$ and floating $V_{f}$ potentials. $I_{\text {el-magn }}=0$ A. Solid lines: $p=5 \mathrm{mTorr}$, $I=4 \mathrm{~A}, U=323 \mathrm{~V}$. Dot lines: $p=2 \mathrm{mTorr}, I=4 \mathrm{~A}, U=370$ V.

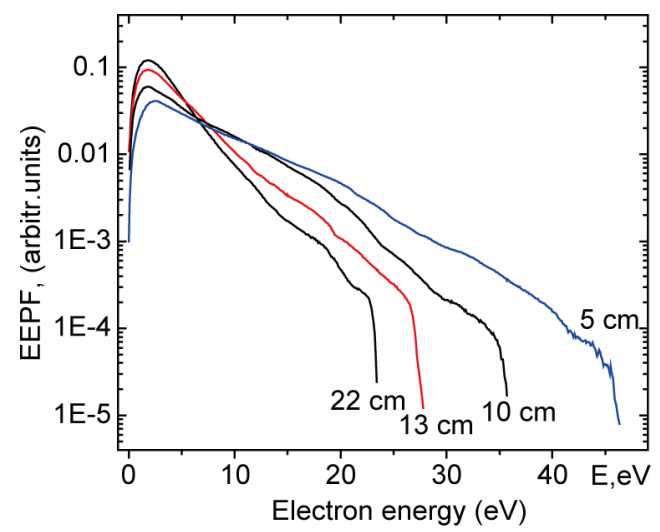

Figure 7. Normalized electron energy probability function on the discharge axis. $p=5 \mathrm{mTorr}, W=1.3 \mathrm{kWatt}$.

energy probability function (EEPF) obtained by dividing the electron energy distribution function (EEDF) by $\sqrt{E}$. This function is convenient because, for a Maxwellian distribution, its logarithm depends linearly on the electron energy [13]. As can be seen from the EEPF plots the energy distributions roughly agree with Maxwellian up to $20 \mathrm{eV}$. The high plasma density provides a strong Maxwelliziing effect due to electron-electron collisions. Be- 
ginning from $20 \mathrm{eV}$ there is a depletion of EEDF due to the inelastic (excitation and ionization) electron collisions with argon atoms. A number of high energy electrons decreases with an increase of distance from cathode. Nevertheless there are many electrons which are able to ionize the copper atoms (the $\mathrm{Cu}$ ionization energy is equal to $7.72 \mathrm{eV}$ ).

The optical emission spectroscopy of $\mathrm{Ar} / \mathrm{Cu}$ plasma was performed as a function of a power and pressure. A typical emission spectrum of wavelength between 210 and $830 \mathrm{~nm}$ from $\mathrm{Cu} / \mathrm{Ar}$ plasma is shown in Figure 8 (the intensities of the resonance lines $\mathrm{Cu} 324.7$ and $\mathrm{Cu} 327.4 \mathrm{~nm}$ are reduced by 5 times).

The spectral line intensity $I\left(v_{i j}\right)$ in optically thin plasma is related to the density atoms in the excited state $\left[X^{*}\right]$ by $[14]$ :

$$
I\left(v_{i j}\right)=c\left(v_{i j}\right) h v\left[X^{*}\right] A_{i j}=K^{v}\left[X^{*}\right],
$$

where $K^{v}$ is a constant specific to each emitted line frequency $v, c\left(v_{i j}\right)$ is the spectral response of the monochromator and detector, $h$-Plank's constant. $\sum_{j} A_{i j}$ represents the sum of all radiative deexcitation frequencies from upper level $i$ to lower level $j$. At higher electron
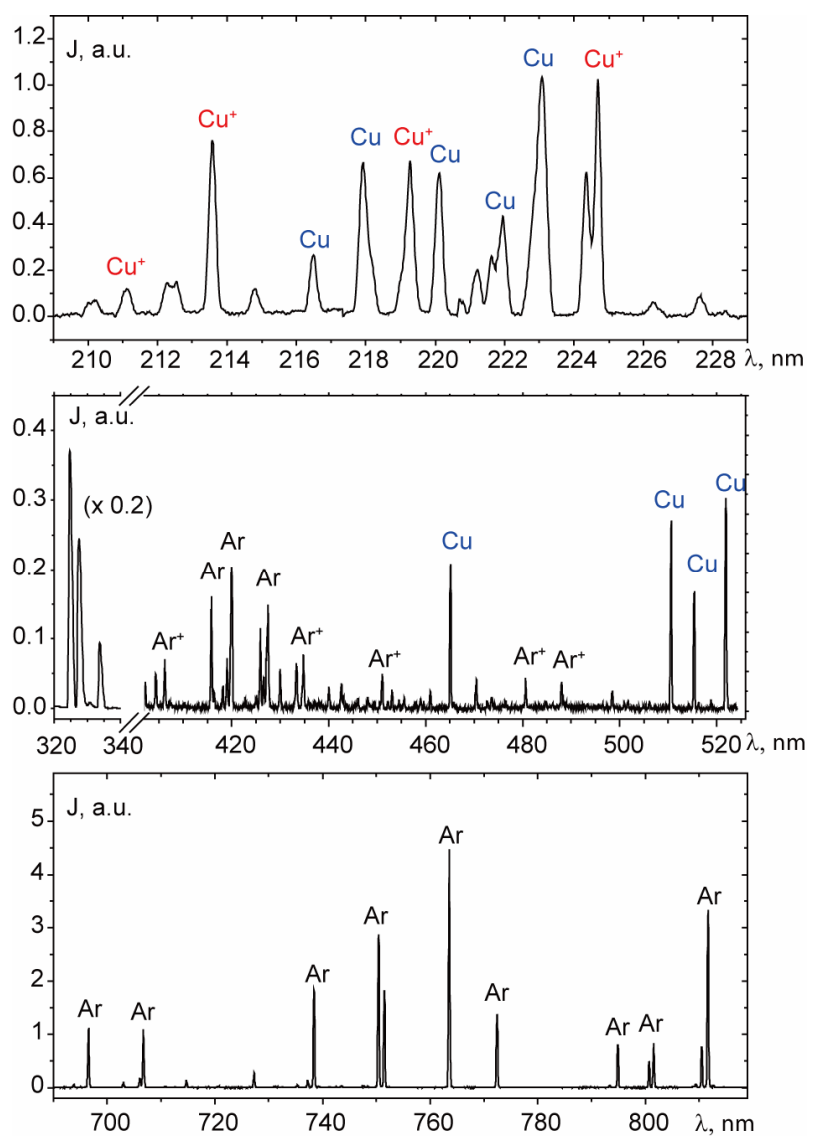

Figure 8. An optical emission spectrum from $\mathrm{Ar} / \mathrm{Cu}$ plasma at wavelength between 210 and $830 \mathrm{~nm}$. $p=5 \mathrm{mTorr}, I_{\mathrm{el}-\mathrm{mag}}=$ $-0.4 \mathrm{~A}, W=3.36 \mathrm{~kW}$. density $\left(>10^{11} \mathrm{~cm}^{-3}\right)$ the electron impact excitation and ionization dominates the Penning mechanism $[3,4]$. Under high electron density conditions collisions decrease the metastable lifetime and therefore we ignore this mechanism.

Then the density of a radiative upper state only populated by electronic collisions depends on the electronic density $n_{e}$

$$
\left[X^{*}\right]=\frac{[X] n_{e} C^{i}}{\sum_{j} A_{i j}+n_{e} C_{i}},
$$

where $C^{i}$ and $C_{i}$ are the production and destruction rates of upper state and $[X]$ density of lower state. For neutral and ionic copper and argon, the radiative loss frequency $\sum_{j} A_{i j}$ is about $10^{7}-10^{8} \mathrm{~s}^{-1}$. According to [15], the loss frequency first excited state of $\mathrm{Ar}$ and $\mathrm{Cu}$ by electronic collision is one order less for $n_{e}<10^{12} \mathrm{~cm}^{-3}$. We therefore assumed that the losses by spontaneous photon emission were dominant process compared with electronic impact loss. Then Equation (2) is written as

$$
I\left(v_{i j}\right)=K^{v} \frac{n_{e}[X] C^{i}}{\sum_{j} A_{i j}}=K_{i j} n_{e}[X] C^{i},
$$

The rate coefficient for electron excitation $C^{i}$ depends on the $E E D F$ and the spectral line intensity is:

$$
I\left(v_{i j}\right)=K_{i j} n_{e}[X] \int_{E_{t}}^{\infty} \sigma v f(E) \mathrm{d} E=K_{i j} n_{e}[X] k\left(T_{e}\right),
$$

where $\sigma$ is the velocity-dependent cross section for electron impact excitation, $f(E)$ is the electron energy distribution function, $v$ is the electron velocity, $E$ and $E_{t}$ represent the electron energy and the excitation threshold energy. For a Maxwellian distribution of electron energies, the integral in Equation (5) is a function of electron temperature represented by an electron temperature dependent rate constant $k\left(T_{e}\right)$. There are two processes, which have the opposite effect on the emission intensity when magnetron power rises. We suppose that rarefaction by buffer gas heating compensates a little growth in electron temperature with increasing discharge power, as probe measurements show that the EEDF depends weakly on a power. Then the emission intensities from both $\mathrm{Cu}$ neutrals and $\mathrm{Cu}^{+}$ions are proportional to the density of the species. The ratio of the emission intensities from $\mathrm{Cu}$ neutral and $\mathrm{Cu}$ ion lines at a constant argon pressure will be proportional to the degree of $\mathrm{Cu}$ ionization. Figure 9 compares the emitted intensities for $\mathrm{Cu}$ neutral $(216 \mathrm{~nm})$ and $\mathrm{Cu}^{+}$ion $(213.6 \mathrm{~nm})$ lines versus discharge power for argon pressure 10 mTorr. Since these lines are close to each other, the photomultiplier sensitivity is equal for them. The emitted intensity from $\mathrm{Cu}$ atom exceeds intensity from $\mathrm{Cu}^{+}$for power less than $1 \mathrm{~kW}$. With increasing power the situation is reversed 


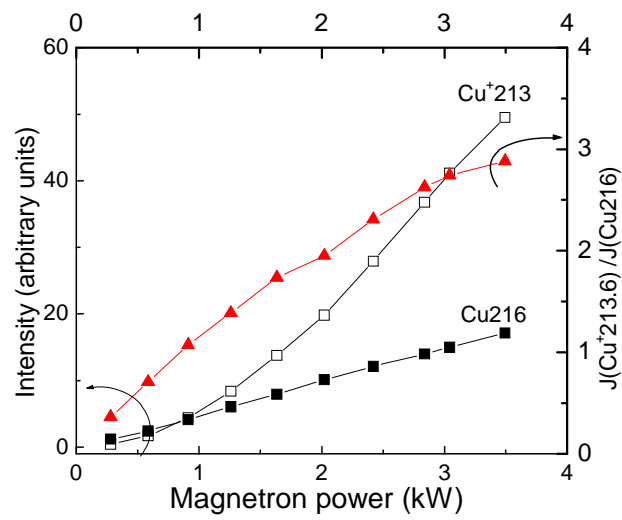

Figure 9. The optical emission intensities from $\mathrm{Cu}$ ion lines $(213.6 \mathrm{~nm})$ and $\mathrm{Cu}$ neutral $(216.5 \mathrm{~nm})$ at $10 \mathrm{mTorr}$ vs discharge power.

and at discharge power of $3.5 \mathrm{~kW}$ the ion emission intensity is about three times higher atom intensity. Thus an increase of electron density with power rise causes effective ionization of the sputtered $\mathrm{Cu}$ atoms.

Figure 10 shows the normalized intensities of $\mathrm{Ar}$ and $\mathrm{Cu}$ spectral lines as function of a magnetron power (intensity at $W=0.9 \mathrm{~kW}$ is accepted for 1) in logarithmic coordinates (numbers within parenthesis show the degree of dependence). These data do not depend on the spectral sensitivity of PMT. It is seen that intensities of radiation of argon and copper atoms increase nearly linear with power. The intensities of argon and copper ions are proportional to about square of the magnetron power. As already noted, the EEDF depends weakly on a power. Therefore dependence of intensity emission is determined mainly by the electron density.

For simplicity, we apply a rather rough assumption that the influence of metastable states is small. Then the following mechanisms are considered for the creation of excited states of argon ions:

$$
\begin{aligned}
& e+\mathrm{Ar} \rightarrow \mathrm{Ar}^{+^{*}}+e+e \quad k_{e, \mathrm{Ar}}^{+^{*}}, \\
& e+\mathrm{Ar}^{+} \rightarrow \mathrm{Ar}^{+^{*}}+e \quad k_{e, \mathrm{Ar}^{+}}^{+^{*}},
\end{aligned}
$$

where $k_{e, \mathrm{Ar}}^{+*}, k_{e, \mathrm{Ar}^{+}}^{+^{*}}$ are the rate coefficients. The excited argon ions density is expressed by

$$
\left[\mathrm{Ar}^{+*}\right]_{\text {atom }}=n_{e}[\operatorname{Ar}]\left(\frac{k_{e, \mathrm{Ar}}^{+*}}{\sum_{j} A_{i j}^{\mathrm{Ar}^{+*}}}\right)=n_{e}[\operatorname{Ar}] C_{+*}^{\mathrm{atom}},
$$

if the electron collides with the atom (see (6)), and

$$
\left[\mathrm{Ar}^{+*}\right]_{\mathrm{ion}}=n_{e}\left[\mathrm{Ar}^{+}\right]\left(\frac{k_{e, \mathrm{Ar}^{+}}^{+*}}{\sum_{j} A_{i j}^{\mathrm{Ar}^{* *}}}\right)=n_{e}\left[\mathrm{Ar}^{+}\right] C_{+*}^{\mathrm{ion}},
$$

if the electron collides with the ion. Here $[\mathrm{Ar}],\left[\mathrm{Ar}^{+}\right]$are atom and ion densities in the ground state respectively.

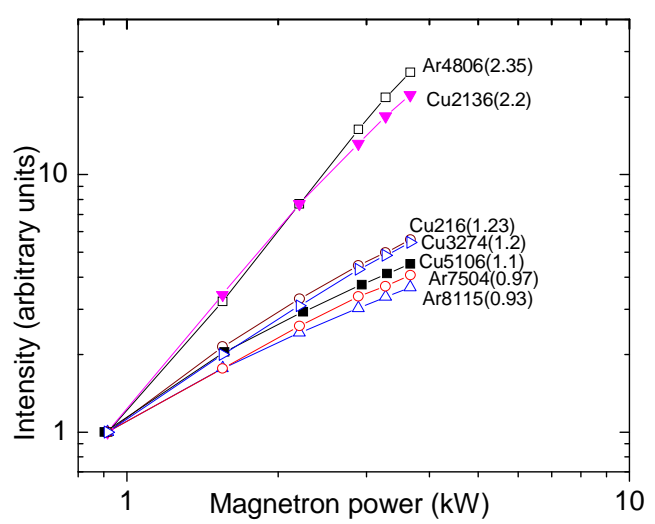

Figure 10. Normalized intensities of $\mathrm{Ar}$ and $\mathrm{Cu}$ spectral lines as function of a magnetron power, $I_{\mathrm{el}}=-0.5 \mathrm{~A}, 10$ mTorr.

The main creation mechanism for argon ions in the ground state is an electronic collision, with the rate coefficient $k_{e, \mathrm{Ar}}^{+}$:

$$
e+\mathrm{Ar} \rightarrow \mathrm{Ar}^{+}+e+e \quad k_{e, \mathrm{Ar}}^{+}
$$

Density of non-radiative species are defined by the losses in electronic collisions and diffusion to the reactor walls. Then in stationary state the density of argon ions in the ground state is given by [15]:

$$
\left[\mathrm{Ar}^{+}\right]=n_{e}[\mathrm{Ar}]\left(\frac{k_{e, \mathrm{Ar}}^{+}}{v_{\mathrm{Ar}^{+}}^{D}+n_{e} k_{e,+}^{+*}}\right),
$$

where $v_{A r^{+}}^{D}$ and $n_{e} k_{e,+}^{+^{*}}$ are the loss frequencies of the argon ion by diffusion and by electron collisions respectively. If the diffusion term is much less than the collision term, then the following expression is obtained from (11):

$$
\left[\mathrm{Ar}^{+}\right]_{\mathrm{coll}}=\frac{[\mathrm{Ar}] k_{e, \mathrm{Ar}}^{+}}{k_{e,+}^{+^{*}}}=[\mathrm{Ar}] C_{+}^{\mathrm{coll}}
$$

Otherwise we have:

$$
\left[\mathrm{Ar}^{+}\right]_{\mathrm{diff}}=\frac{n_{e}[\mathrm{Ar}] k_{e, \mathrm{Ar}}^{+}}{v_{\mathrm{Ar}^{+}}^{D}}=n_{e}[\mathrm{Ar}] C_{+}^{\mathrm{diff}},
$$

where the constants $C_{+}^{\text {coll }}$ and $C_{+}^{\text {diff }}$ are defined as the ratio between the creation coefficient and the loss coefficient. At constant pressure they are assumed do not depend on discharge power.

Using (4) the emitted intensity of the Ar ion is

$$
I\left(\mathrm{Ar}^{+^{*}}\right)=K_{i j}^{\mathrm{Ar}^{+}} n_{e}[\mathrm{Ar}] C_{+^{*}}^{\mathrm{atom}},
$$

if argon ion excited from the argon neutral (see (8)). When argon ion excited from the ion ground state and minor diffusion (see (9) and (12)), the intensity can be written: 


$$
\begin{aligned}
I\left(\mathrm{Ar}^{+^{*}}\right) & =K^{v}\left[\mathrm{Ar}^{+^{*}}\right]_{\text {ion }}=K^{v} n_{e}\left[\mathrm{Ar}^{+}\right]_{\text {coll }} C_{+*}^{\text {ion }} \\
& =K^{v} n_{e} C_{+*}^{\text {ion }}[\mathrm{Ar}] C_{+}^{\text {coll }}
\end{aligned}
$$

If argon ion excited from the ion ground state and dominant diffusion (see (13)) we have:

$$
\begin{aligned}
I\left(\mathrm{Ar}^{+^{*}}\right) & =K^{v}\left[\mathrm{Ar}^{+^{*}}\right]_{\text {ion }}=K^{v} n_{e}\left[\mathrm{Ar}^{+}\right]_{\text {diff }} C_{+*}^{\text {ion }} \\
& =K^{v} n_{e} C_{+^{*}}^{\text {ion }} n_{e}[\mathrm{Ar}] C_{+}^{\text {coll }}
\end{aligned}
$$

It is seen from the previous expressions, that the emission intensity is related to the electronic density, which is about linearly proportional to the magnetron power.

The intensities of argon ions are proportional to about square of the magnetron power as it follows from Figure 9. This implies that the main losses for argon ions are by diffusion at $20 \mathrm{~cm}$ according to expression (16).

Similar equations can be obtained for copper atom and ion lines. The copper atom density in the ground state at the steady state is given by:

$$
[\mathrm{Cu}]=\frac{\gamma_{\mathrm{Cu}}\left[\mathrm{Ar}^{+}\right]}{v_{\mathrm{Cu}}^{D}+n_{e} k_{e, \mathrm{Cu}}^{+}},
$$

where $\gamma_{\mathrm{Cu}}$ is the sputtering coefficient and $k_{e, \mathrm{Cu}}^{+}$is the destructive rate by ionization in the following reaction:

$$
e+\mathrm{Cu} \rightarrow \mathrm{Cu}^{+}+e+e
$$

If copper excited states are supposed to be created mainly by the electron impact on the copper ground state

$$
e+\mathrm{Cu} \rightarrow \mathrm{Cu}^{*}+e,
$$

Then the line intensity can be expressed versus electron density as:

$$
I\left(\mathrm{Cu}^{*}\right) \propto n_{e}[\mathrm{Cu}] \propto \frac{n_{e} \gamma_{\mathrm{Cu}}\left[\mathrm{Ar}^{+}\right]_{\mathrm{diff}}}{v_{\mathrm{Cu}}^{D}+n_{e} k_{e, \mathrm{Cu}}^{+}} \propto \frac{n_{e}^{2} \gamma_{\mathrm{Cu}}[\mathrm{Ar}]}{v_{\mathrm{Cu}}^{D}+n_{e} k_{e, \mathrm{Cu}}^{+}}
$$

Here we used Equations (13) and (17). If diffusion term $v_{\mathrm{Cu}}^{D}$ is much less than collision term $n_{e} k_{e, \mathrm{Cu}}^{+}$,

$$
v_{\mathrm{Cu}}^{D} \ll n_{e} k_{e, \mathrm{Cu}}^{+}
$$

we obtain:

$$
I\left(\mathrm{Cu}^{*}\right) \propto n_{e}
$$

Figure 10 shows that emission intensity of copper atoms increases linearly with a slope 1 . Thus, the proposed kinetic scheme with the expressions (13) and (17) explains the observed behavior of $\mathrm{Cu}$ line intensities, indicating that copper atoms were lost due to the ionization by electron collisions at a distance of $20 \mathrm{~cm}$ from magnetron.

Another argument in favor of this conclusion is the results of experiments on the absorption of resonance lines of copper. Figure 11 shows the absorption coefficient $\mathrm{A}$ of the resonance $\mathrm{Cu}$ line $324.7 \mathrm{~nm}$, obtained at a

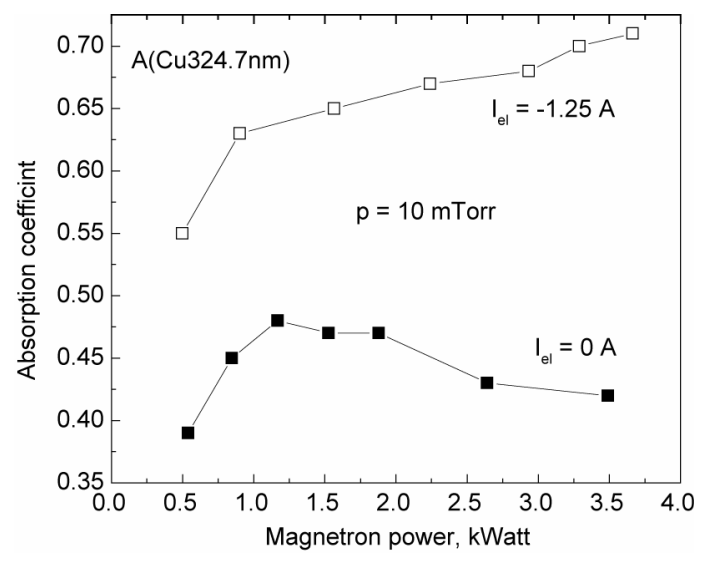

Figure 11. Absorption coefficient $A$ of the $\mathrm{Cu}$ spectral line $324.7 \mathrm{~nm}$ as function of magnetron power.

distance of $20 \mathrm{~cm}$ vs magnetron power. $A=1-\frac{I_{L+P}-I_{P}}{I_{L}}$, where $I_{L}$ is the light intensity of the lamp with hollow cathode, $I_{P}$ is intensity of $\mathrm{Cu}$ atoms in plasma, when lamp off and $I_{L+P}$ is the intensity, measured with lamp and plasma on. When current of electromagnet $I_{\mathrm{el}}=$ -1.25 A plasma density at this distance is low (see Figure 4) and ionization of $\mathrm{Cu}$ atoms also small. Density of sputtered $\mathrm{Cu}$ atoms increases with growing power and coefficient A grows. When $I_{\mathrm{el}}=-0.6$ A plasma density downstream the magnetron is great and increases with magnetron power. The ionization of $\mathrm{Cu}$ atoms grows also, density of $\mathrm{Cu}$ atoms decreases and coefficient $A$ falls. Recall that value of the sputtered $\mathrm{Cu}$ atoms inside of the cathode is about the same in both cases. These measurements confirm our conclusion that the ionization of $\mathrm{Cu}$ atoms is dominant loss term at a distance of $20 \mathrm{~cm}$ from magnetron. Detailed description of these measurements is beyond scope of this article.

For the emission intensity of the copper ion we consider two-step mechanism:

$$
\mathrm{Cu}^{+}+e \rightarrow \mathrm{Cu}^{+*}+e,
$$

where $\mathrm{Cu}^{+}$is produced by reaction:

$$
\mathrm{Cu}+e \rightarrow \mathrm{Cu}^{+}+e+e
$$

Then

$$
I\left(\mathrm{Cu}^{+^{*}}\right) \propto n_{e}\left[\mathrm{Cu}^{+}\right]
$$

The copper ion density in the ground state is given by analogy to (11):

$$
\left[\mathrm{Cu}^{+}\right]=n_{e}[\mathrm{Cu}]\left(\frac{k_{e, \mathrm{Cu}}^{+}}{v_{\mathrm{Cu}^{+}}^{D}+n_{e} k_{e, \mathrm{Cu}^{+}}^{+}}\right),
$$

Using condition (21) and Equations (17) and (26) it can be deduce 


$$
\begin{aligned}
& I\left(\mathrm{Cu}^{+^{*}}\right) \propto n_{e}\left[\mathrm{Cu}^{+}\right] \propto \frac{n_{e}^{2}[\mathrm{Cu}]}{\left(v_{\mathrm{Cu}^{+}}^{D}+n_{e} k_{e, \mathrm{Cu}^{+}}^{+}\right)} \\
& \propto \frac{\gamma_{\mathrm{Cu}} n_{e}^{2}\left[\mathrm{Ar}^{+}\right]_{\mathrm{diff}}}{\left(v_{\mathrm{Cu}^{+}}^{D}+n_{e} k_{e, \mathrm{Cu}^{+}}^{+}\right)\left(v_{\mathrm{Cu}}^{D}+n_{e} k_{e, \mathrm{Cu}}^{+}\right)} \\
& \propto \frac{\gamma_{\mathrm{Cu}} n_{e}^{3}[\mathrm{Ar}]}{\left(v_{\mathrm{Cu}^{+}}^{D}+n_{e} k_{e, \mathrm{Cu}^{+}}^{+}\right) n_{e} k_{e, \mathrm{Cu}}^{+}} \propto \frac{n_{e}^{2}[\mathrm{Ar}]}{\left(v_{\mathrm{Cu}^{+}}^{D}+n_{e} k_{e, \mathrm{Cu}^{+}}^{+}\right) k_{e, \mathrm{Cu}}^{+}}
\end{aligned}
$$

The copper ion diffusion term $v_{\mathrm{Cu}^{+}}^{D}$ is much more than total destructive (including double ionization) term $n_{e} k_{e, \mathrm{Cu}^{+}}^{+}$. So that

$$
I\left(\mathrm{Cu}^{+*}\right) \propto n_{e}^{2}
$$

Thus, from the intensity variation of $\mathrm{Cu}$ and $\mathrm{Cu}^{+}$spectral lines with magnetron power, it is deduced that the dominant loss term are electron ionization for the copper atoms and diffusion for the copper ions.

In our model we neglect the influence of metastable states of $\mathrm{Cu}$ and $\mathrm{Ar}$ atoms. Our measurements of the absorption coefficient of the line Cu510.6 nm shown that density of the metastable level ${ }^{2} \mathrm{D}_{5 / 2}$ is of an order less than density of the ground level. From the absorption coefficient of Ar696.5 and Ar811.5 nm lines we calculated density of metastable Ar level $s_{5}$ (Pashen notation). The Ar metastable state density was found in the range of $10^{10}-10^{11} \mathrm{~cm}^{-3}$. Note that these data were obtained at a distance of $20 \mathrm{~cm}$ from the target. Data on metastable Ar atoms are in good agreement with the results obtained in a high-density plasma discharges [16-18]. We also did not account for Penning ionization of $\mathrm{Cu}$ and $\mathrm{Ar}$ atoms. Due to these factors, the experimental intensities of $\mathrm{Ar}^{+}$, $\mathrm{Cu}$ and $\mathrm{Cu}^{+}$increase faster. However, these differences are small, indicating that these processes make a small contribution to ionization process.

The carried out experiments have shown, that magnetron hollow cathode discharge allows to receive at pressure in some mTorr plasma density more than $10^{11} \mathrm{~cm}^{-3}$ at a distance in tens $\mathrm{cm}$. The high plasma density created in the big volume, increases probability of ionization of the sprayed atoms of a target. The stream of ions of the target, controlled by an electric field near a substrate, enables to deposit a highly conformal film on structures of the complex form. The size, uniformity, a degree of ionization of a stream of plasma can be supervised by the appropriate choice of power, pressure, magnitude and configuration of a magnetic field.

\section{Conclusion}

Langmuir probe and optical emission spectroscopy measurements were used to study of plasma characteristics and $\mathrm{Cu}$ ionization in $\mathrm{HCM}$ discharge. The pressure range is $0.5-10 \mathrm{mTorr}$ with $1-5 \mathrm{~kW}$ discharge power. Variation in the plasma parameters such as electron densities and temperatures, electron energy distribution function, plasma space and floating potentials as a function of the position, pressure and power in the growth chamber were measured in detail. The optical emission spectroscopy at a distance of $20 \mathrm{~cm}$ from magnetron shows strong increase of the intensity ratio from $\mathrm{Cu}^{+}$ion and $\mathrm{Cu}$ neutral lines with the power. These measurements indicated large downstream ionization of sputtered copper atoms. From the intensity variation of argon and copper atoms and ions spectral lines with magnetron power, it is deduced that the main creation mechanism for argon and copper ions is an electronic collision from the ground state and the dominant loss terms are electron ionization for copper atoms and diffusion for the ions.

\section{REFERENCES}

[1] E. Klawuhn, G. C. D'Couto, K. A. Ashtiani, P. Rymer, M. A. Biberger and K. B. Levy, "Ionized Physical-Vapor Deposition Using a Hollow-Cathode Magnetron Source for Advanced Metallization," Journal of Vacuum Science \& Technology, Vol. 18A, No. 4, 2000, pp. 1546-1549.

[2] V. Vyas and M. J. Kushner, "Scaling of Hollow Cathode Magnetrons for Ionized Metal Physical Vapor Deposition," Journal of Vacuum Science \& Technology, Vol. 24A, No. 5, 2006, pp. 1955-1969.

[3] J. Hopwood and F. Qian, "Mechanism for Highly Ionized Magnetron Sputtering," Journal of Applied Physics, Vol. 78, No. 2, 1995, pp. 758-765. doi:10.1063/1.360334

[4] J. Hopwood, "Ionized Physical Vapor Deposition of Integrated Circuit Interconnects," Physics of Plasmas, Vol. 5, No. 5, 1998, pp. 1624-1631. doi:10.1063/1.872829

[5] S. M. Rossnagel, "Thin Film Deposition with Physical Vapor Deposition and Related Technologies," Journal of Applied Physics, Vol. 21, No. 5, 2003, pp. 74-87. doi:10.1116/1.1600450

[6] K. Ostrikov and A. B. Mutphy, "Plasma-Aided Nanofabrication: Where Is the Cutting Edge?" Journal of Physics D: Applied Physics, Vol. 40, No. 8, 2007, pp. 2223-2241. doi:10.1088/0022-3727/40/8/S01

[7] A. Anders, "Metal Plasmas for the Fabrication of Nanostructures," Journal of Physics D: Applied Physics, Vol. 40, No. 8, 2007, pp. 2272-2284. doi:10.1088/0022-3727/40/8/S06

[8] S. M. Gorbatkin and S. M. Rossnagel, "Cu Metallization Using a Permanent Magnet ECR Microwave Plasma/ Sputtering Hybrid System," Journal of Vacuum Science \& Technology, Vol. 14B, No. 3, 1996, pp. 1853-1859.

[9] L. Meng, R. Raju, R. Flauta, H. Shin and D. N. Ruzic, "In Situ Plasma Diagnostics Study of a Commercial HighPower Hollow Cathode Magnetron Deposition Tool," Journal of Vacuum Science \& Technology, Vol. 28A, No. 1, 2010, pp. 112-118.

[10] L. Wu, E. Ko, A. Dulkin, K. J. Park, S. Fields, K. Leeser, L. Meng and D. N. Ruzic, "Flux Energy Analysis of Spe- 
cies in Hollow Cathode Magnetron Ionize Physical Vapor Deposition of Copper," Review of Scientific Instruments, Vol. 81, No. 12, 2010, Article ID: 123502.

[11] J. G. Laframboise "Theory of Spherical and Cylindrical Langmuir Probes in a Collisionless Maxwellian Plasma at Rest," University of Toronto, Toronto, 1966.

[12] M. Mausbach, "Parametrization of the Laframboise Theory for Cylindrical Langmuir Probe Analysis," Journal of Vacuum Science \& Technology, Vol. 15A, No. 6, 1997, pp. 2923-2929.

[13] M. A. Lieberman and A. J. Lichtenberg, "Principles of Plasma Discharges and Materials Processing," Wiley, New York, 1994.

[14] R. W. McWhirter, "Spectral Intensities," In: R. H. Huddlestone and S. L. Leonard, Eds., Plasma Diagnostic Techniques, Academic, New York, 1965, pp. 165-217.

[15] C. Nouvellon, S. Konstantinidis, J. P. Dauchot, M. Wautelet, P. Y. Jouan, A. Ricard and M. Hecq, "Emission
Spectrometry Diagnostic of Sputtered Titanium in Magnetron Amplified Discharge," Journal of Applied Physics, Vol. 92, No. 1, 2002, pp. 32-36. doi:10.1063/1.1481780

[16] J. B. Boffard, R. O. Jung, Ch. C. Lin and A. E. Wendt, "Measurement of Metastablt and Resonance Level Densities in Rare-Gas Plasmas by Optical Emission Spectroscopy," Plasma Sources Science and Technology, Vol. 18, No. 3, 2009, pp. 1-11. doi:10.1088/0963-0252/18/3/035017

[17] G. A. Hebner, "Spatially Resolved, Exited State Densities and Neutral and Ion Temperatures in Inductively Coupled Plasmas," Journal of Applied Physics, Vol. 80, No. 5, 1996, pp. 2624-2636. doi:10.1063/1.363178

[18] D. Leonhardt, C. R. Eddy, V. A. Shamamian, R. F. Fensler and J. E. Butler, "Argon Metastables in a High Density Processing Plasma," Journal of Applied Physics, Vol. 83, No. 6, 1998, pp. 2971-2978. doi: $10.1063 / 1.367123$ 